\title{
Interpretation of uncommon ECG findings in patients with atrial flutter
}

\author{
Mykhaylo S. Sorokivskyy, Ulyana P. Chernyaha-Royko \\ Lviv National Medical University named after Danylo Halytskyy, \\ Lviv Regional State Clinical Treatment and Diagnostics Cardiology Center, Lviv, Ukraine
}

\begin{abstract}
In this article, several uncommon cases of different atrial flutter (AFL) types are described. Electrocardiogram (ECG), the standard and widespread method of rhythm disturbances detection, sometimes can be insufficient for correct diagnosis in such cases. Different features of atrioventricular (AV) conduction and multiple atrial macro re-entry circles may considerably influence the surface ECG. Another distorting factor of AFL appearance is possibility of ECG elements superposition and overlapping. Some atypical forms of AFL can be poorly visible and sometimes cannot be differentiated from other types of supraventricular arrhythmias on surface 12-lead ECG.
\end{abstract}

(Heart Vessels and Transplantation 2017; 1: doi: 10.24969/2017.6)

\section{Introduction}

Atrial flutter (AFL) is one of the most common supraventricular arrhythmias with macro-reentrant mechanism of atrial activation around an anatomical barrier with regular atrial rate $\geq 240$ beats/min $(1,2)$. Despite of recent advances in invasive electrophysiology, electrocardiography (ECG) remains one of the most effective methods used for rhythm disorders diagnostics. It is still the tool of the first choice for practitioners that largely determines further diagnostics and treatment strategy to be used to manage a patient $(3,4)$. However, proper interpretation of ECG may be rather difficult in patients with AFL and different types of atrial tachycardias (AT). Sometimes we need only atrial mapping for precise diagnosis. Atrial flutter can be described as counterclockwise or clockwise typical (cavotricuspid isthmus (CTI)dependent) and atypical by ECG (2). For clinicians, a key point of ECG diagnostics of typical AFL is to identify a "saw tooth" flutter curve with characteristic F waves in II, III, aVF and V1 leads, which are caused by AFL waves circulating in the right atrium. In 10-30\% of typical AFLs, the reentrant circuit rotates in a clockwise direction around the tricuspid valve, which causes the changing $\mathrm{F}$ waves polarity (1-3, 5). In real clinical practice, the pattern of typical AFL occurs only in $70-80 \%$ of patients $(1,4)$, that is why the diagnostics of atypical AFL requires thorough ECG analysis. Features of atrioventricular (AV) conduction, unusual aberrant ventricular conduction, coexisting with atrial fibrillation (AF) result in diverse ECG picture of AFL (6).

In this article, we examine ECG fragments in patients with different types of AFL, analyze AV conduction and provide a summary of recent approaches and algorithms applied in ECG diagnostics of AFL.

\section{Different conduction patterns in AFL}

In AFL, atrial $\mathrm{F}$ waves usually occur at a rate within 220-350 bpm, although the rate may decrease below $200 \mathrm{bpm}$ or increase to $400 \mathrm{bpm}$. The maximum possibility of AV node conduction rarely exceeds $180-220 \mathrm{bpm}$. This value depends on many factors and decreases with age. While absolute irregularity of RR intervals is characteristic of AF, these intervals may be both regular and irregular in case of AFL. Sometimes AFL involves a particular pattern of AV conduction called "regular irregularity". 


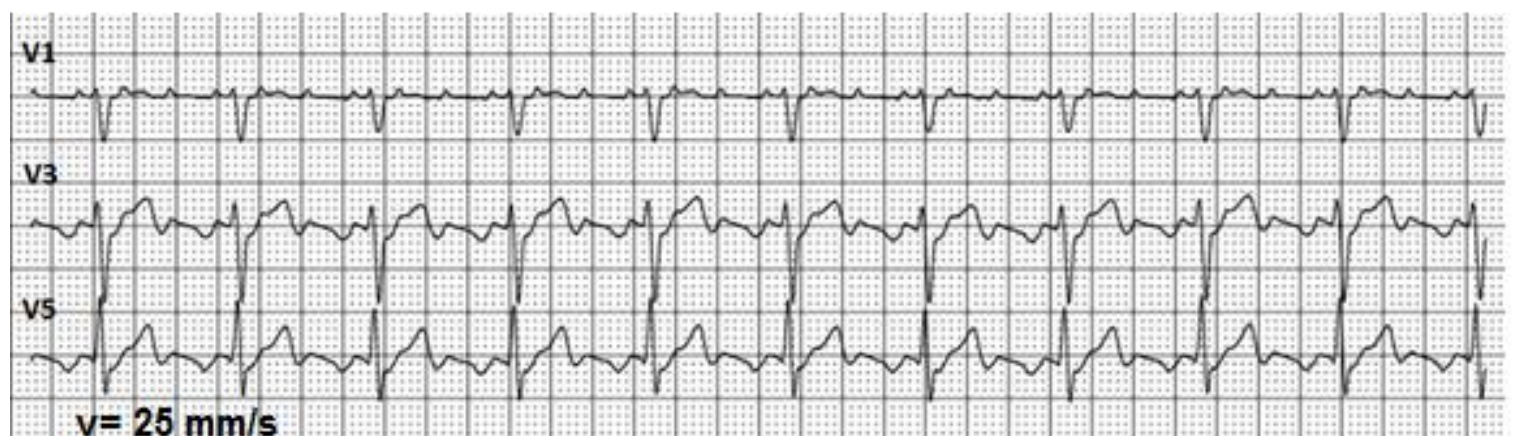

Figure 1. Atrial flutter with 3:1 regular conduction.

For most AFL cases, a ratio of AV conduction can be calculated. Most frequently, AV conduction equals $2: 1$ or $4: 1$, while a 3:1 is less common (Fig. 1). Quite often, a regular 2:1 conduction across the AV node is difficult to interpret because $\mathrm{F}$ waves overlap QRS complexes ant $\mathrm{T}$ waves. Sometimes it makes impossible to make a correct diagnosis using a standard 12-lead ECG. However, the analysis of a long ECG record or Holter monitoring is beneficial in identifying episodes with a decreased or increased atrial rate or episodes of irregular conduction with clearly visible $\mathrm{F}$ waves. This data considerably facilitates the diagnosis. When required, vagal tests or adenosine via intravenous injection can be applied to cause a short time block of conduction in the AV node (7). During this short period, electrical activity of atria becomes clearly visible on ECG.

In some cases, AV node conduction increases due to influence of some factors (activated sympathetic nervous system, hyperthyroidism). On the other side, the use of class I antiarrhythmic drugs is known to slow down the atrial rate while the AV conduction remains the same or increases. This may result in developing AFL with 1:1 AV conduction. The His-Purkinje system cannot properly conduct impulses with rate beyond 200 per minute and aberrant conduction with wide QRS complex occurs (Fig. 2a.). Very high rate and aberrant ventricular complexes often result in a significant reduction of cardiac output and deterioration of patients` systemic hemodynamics. In young patients, having normal function of conduction system such clinical situation can lead to AFL with 1:1 AV conduction with normal ventricular complexes (Fig. 2b).

Obviously, this narrow QRS complex tachycardia must be differentiated from other types of supraventricular tachycardia, such as atrial tachycardia and AV-node tachycardia $(3,10)$. However, rarely AFL occurs with 1:1 conduction: extraordinary high heart rate around $225 \mathrm{bpm}$, and some episodes of irregular ventricular conduction, which can be seen during further observation (Fig.2c.) 


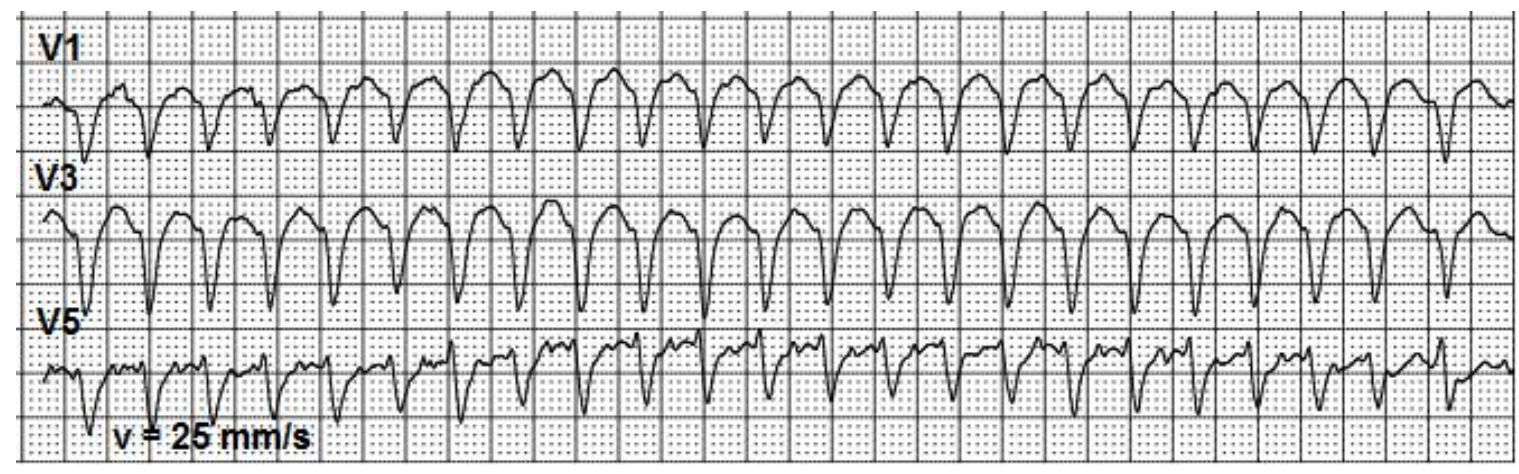

Figure 2a. Atrial flutter with 1:1 atrioventricular conduction and $210 \mathrm{bpm}$ ventricular rate with aberrant ventricular complexes.

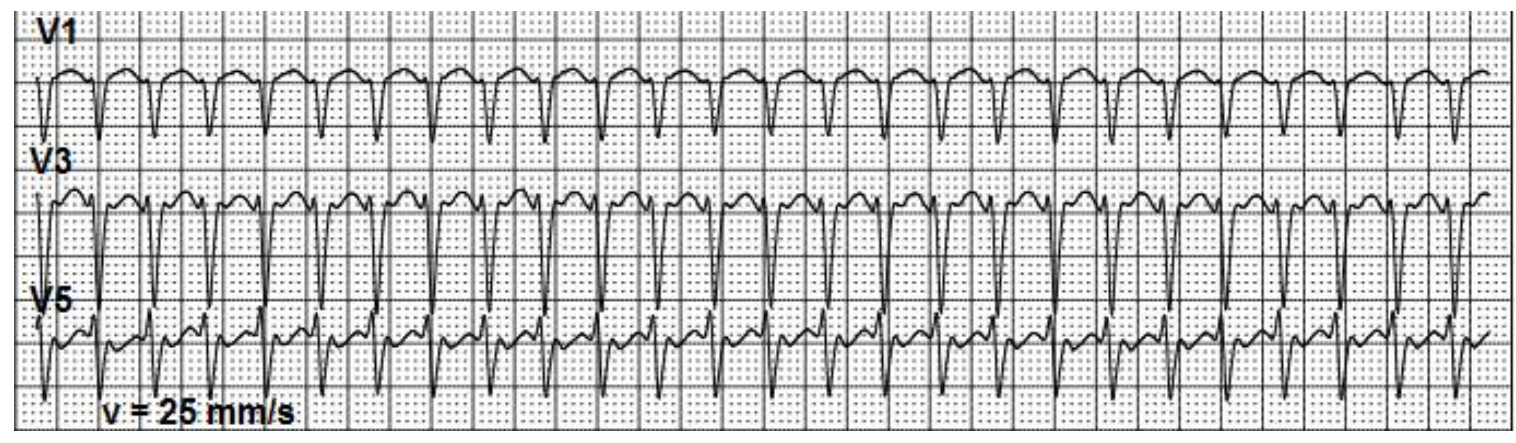

Figure $2 \mathrm{~b}$. Atrial flutter with 1:1 atrioventricular conduction and about $225 \mathrm{bpm}$ ventricular rate and normal ventricular conduction.

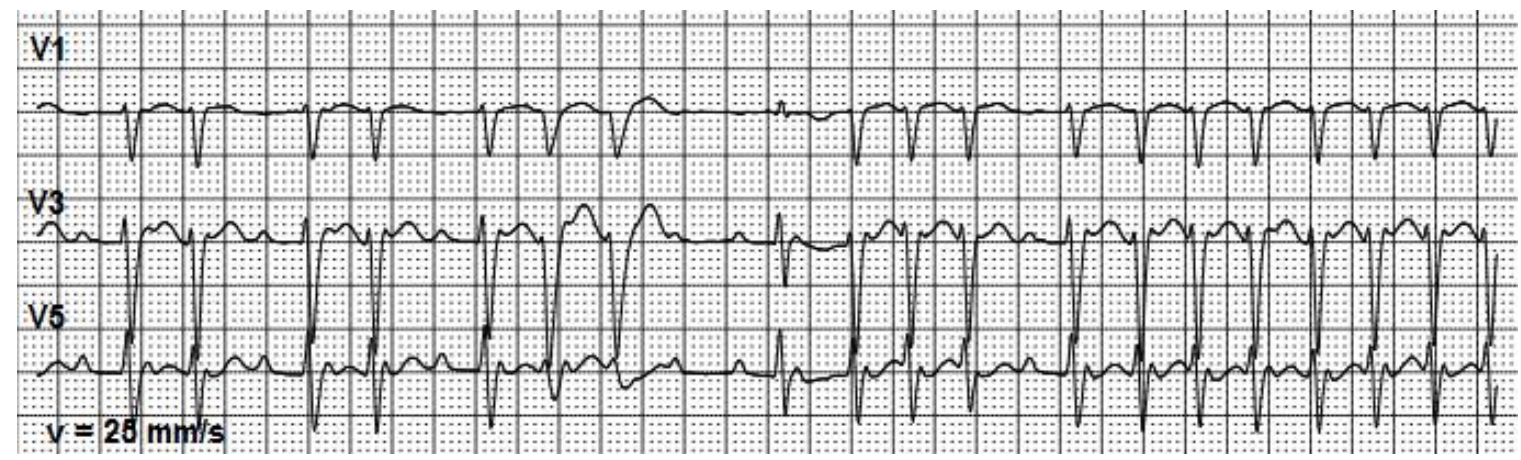

Figure 2c. Atrial flutter with irregular ventricular conduction and episodic aberrant conduction 


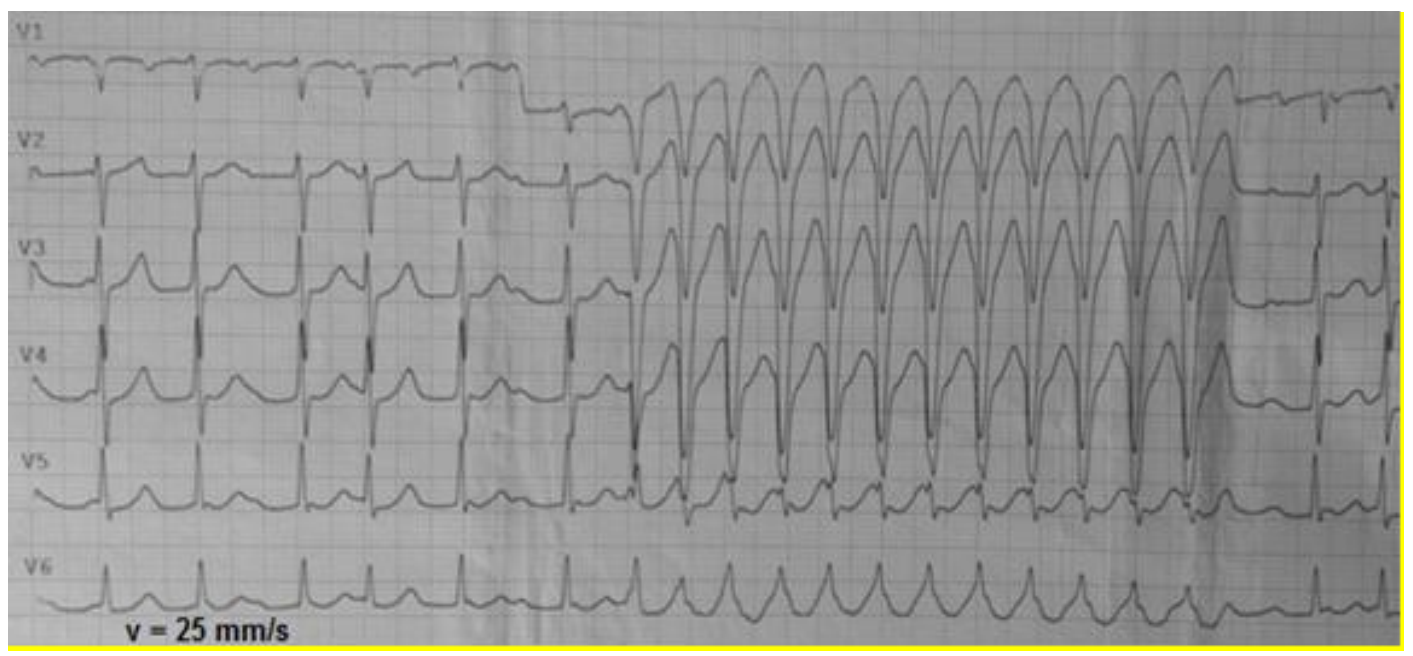

Figure 3. Irregular atrial flutter followed by 1:1 conduction episode with aberrant QRS complexes.

Atrioventricular node conduction can vary. Rapid increase of $\mathrm{AV}$ conduction may significantly accelerate ventricular rate. In such cases, it is difficult to differentiate flutter with $1: 1 \mathrm{AV}$ conduction from ventricular tachycardia. Rate of $\mathrm{F}$ waves on previous ECG fragment with slower AV conduction can help to determine 1:1 atrial flutter as a cause of wide regular tachycardia. (Fig. 3). The beginning of ECG shows that the rate of atrial $F$ waves is equal to the rate of ventricular beats in wide tachycardia.

This fact confirms aberrant conduction of atrial impulses to ventricles with rate at about $170 \mathrm{bpm}$.

Another cause of 1:1 conduction of flutter waves to the ventricles can be an accessory pathway with short refractory period. In this case, impulse passes by AV node and enters ventricles causing wide and regular QRS complexes.

Atrial rate in AFL may drop down to $200 \mathrm{bpm}$, and even to $150 \mathrm{bpm}$ due to antiarrhythmic therapy. The main factors determining atrial rate in atrial flutter are the size of macro re-entry circle and electrophysiological properties of atrial tissues.

Observations from long-term monitoring demonstrated that AFL could spontaneously stop or transform into other forms of supraventricular arrhythmias $(3,4)$. The most often AFL changes to atrial fibrillation $(6,8,9)$; changes between different types of atrial flutter are less common. Figure 4 shows AFL with atrial rate of 175 per minute, regular 2:1 conduction and ventricular rate of $87 \mathrm{bpm}$ spontaneously changing to AFL with atrial rate of 280 per minute, conduction $4: 1$ and ventricular rate $70 \mathrm{bpm}$. The most likely explanation of this phenomenon is a change of the impulse circulation path to shorter one that accelerates the impulse circulation while AV node conduction and ventricular rate changed slightly.

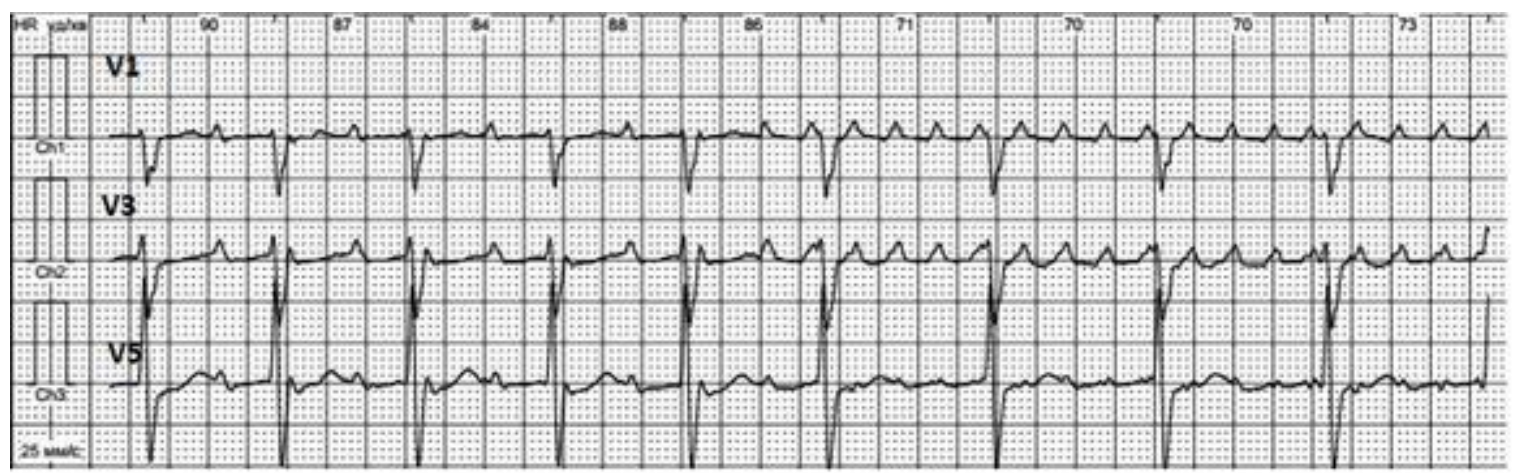

Figure 4. Atrial flutter with 2:1 conduction changes to 4:1 conduction. 


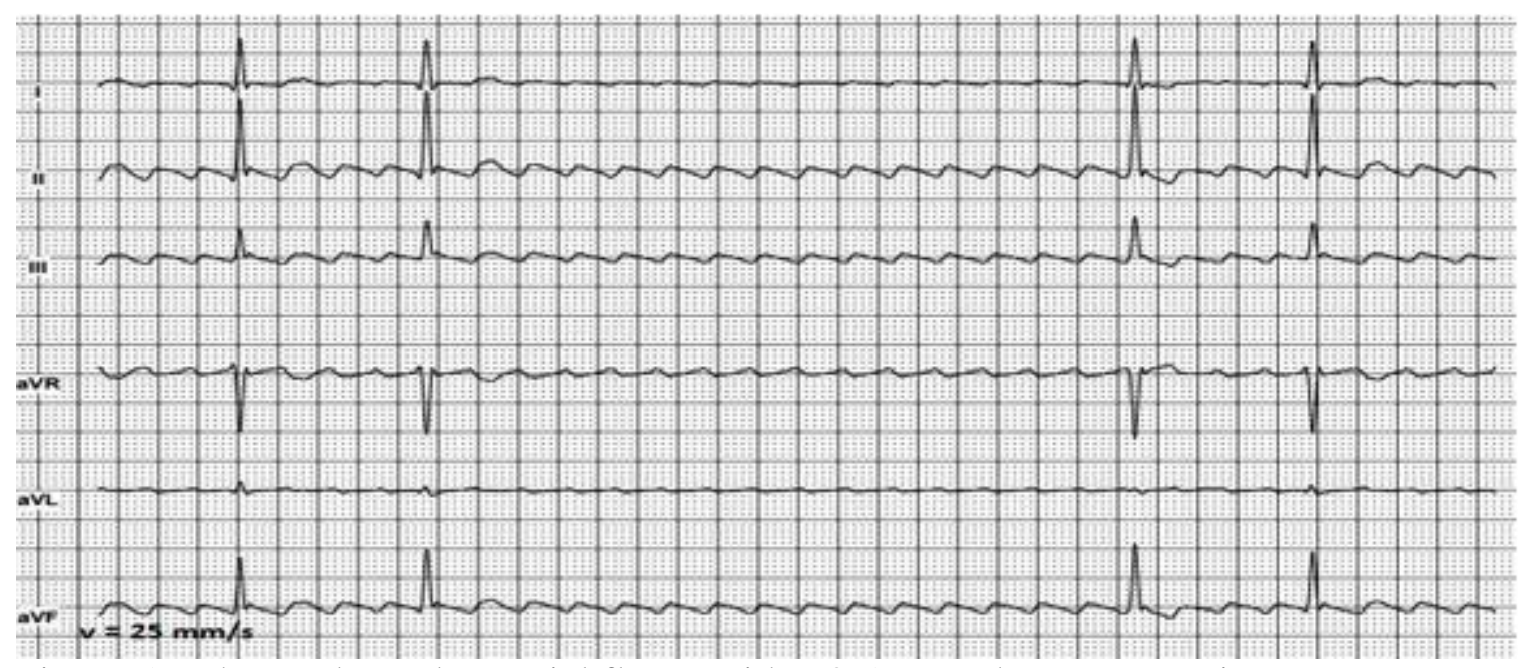

Figure 5. Isthmus-dependent atrial flutter with a 3.5 second asymptomatic pause.

Interpretation of the atrial flutter recordings sometimes is complicated when ECG shows pauses of various duration due to episodically worsening of AV conduction. Figure 5 presents a classical isthmus-dependent AFL with 3.5 seconds pause. Long pauses, which can be seen on ECG, are clinically important signs of impaired AV conduction and may be considered as indication to implantation of the pacemaker. In presented case, the pause was not associated with any symptoms and the patient did not complain of weakness or dizziness, associated with bradycardia.

Asymptomatic pauses shorter than 4-6 seconds during AF/AFL do not require pacemaker implantation. Radiofrequency ablation procedure should be performed due to isthmus-dependent atrial flutter. After sinus rhythm restoration, recurrence of $\mathrm{AV}$ node conduction disturbances is less likely.
Prolonged bradycardia episodes with rhythmic ventricular complexes may also be present during AFL.

ECG pattern shown on Figure 6, may suggest a complete AV-node conduction block, Frederick syndrome. At the same time, QRS complexes of the same morphology, which are conducted by $\mathrm{AV}$-node faster than in established rhythm, can be seen. Therefore, it can be concluded that AV-node conduction is preserved and possibility of complete AV-node block is less likely in this case. The ECG on Figure 6 presents a short episode of AFL with regular 8:1 AV node conduction with further transformation in atrial flutter with irregular conduction. During atrial flutter, a number of impulses entering AV node exceeds its conduction capacity. Therefore, the functional AV block occurs in the atrioventricular node to prevent excessively high contraction rate of ventricles and maintain systemic hemodynamics parameters.

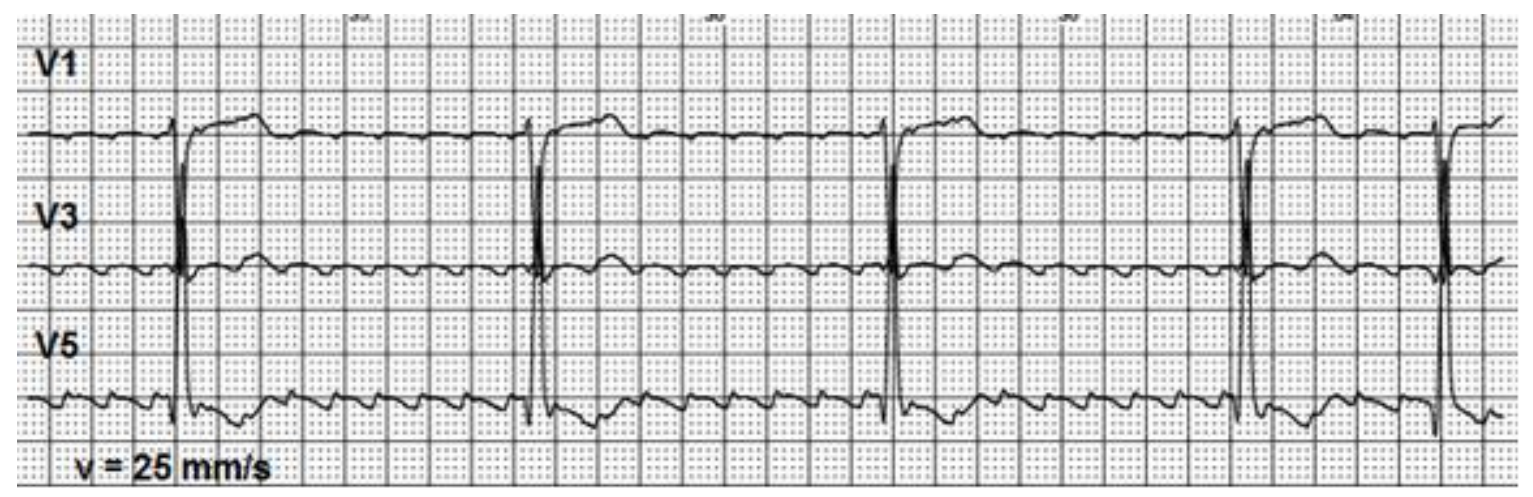

Figure 6. A short episode of 8:1 regular atrioventricular conduction. 


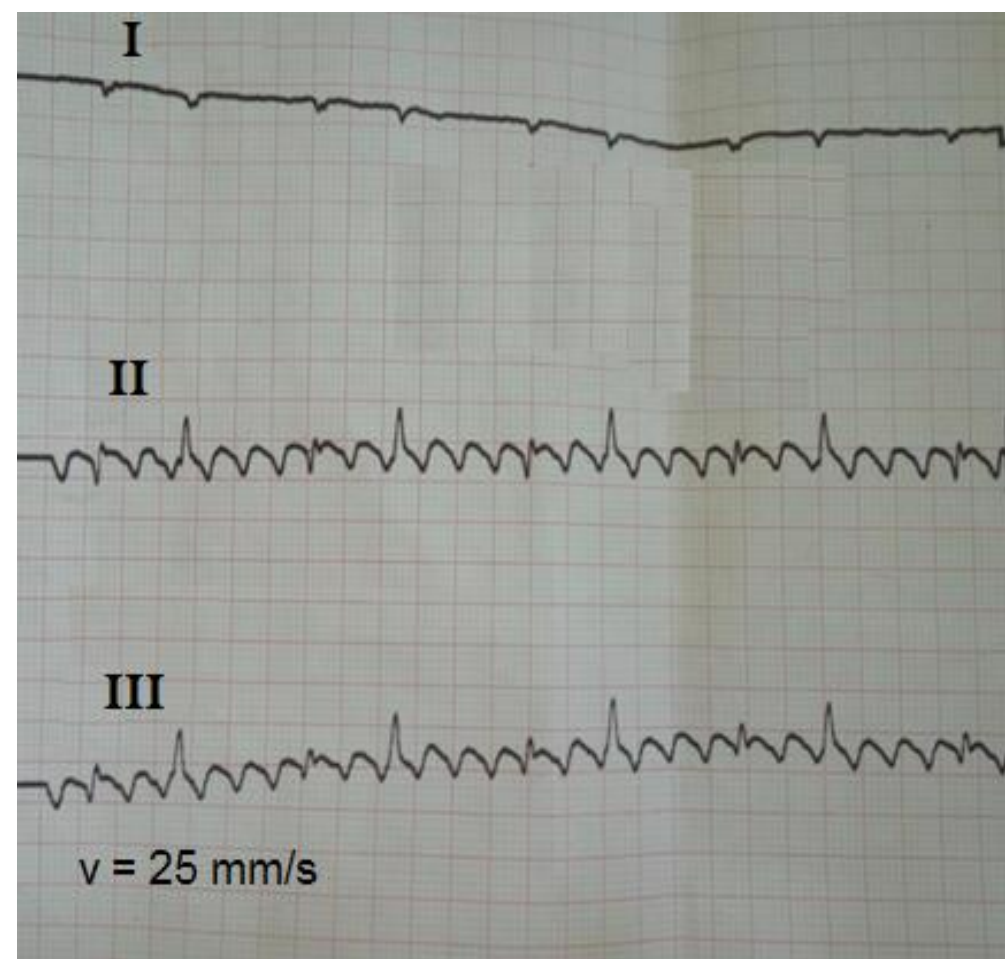

Figure 7a. Atrial flutter with a rare conduction 6:2 type and significant overlapping of QRS complexes and $\mathrm{F}$ waves.

Figure 7a shows a specific multilevel block of AV-node conduction with type A alternating Wenckebach phenomenon (11). The first impulse seems to be normally conducted; the second impulse is blocked in upper level of AV node; the third is conducted with a delay; the fourth is blocked in upper level of AV node; conduction of the fifth is blocked in lower parts of AV junction; and the sixth impulse is not conducted because of blockade in upper level of AV node. ECG on Figure 7a presents a 6:2 node conduction pattern, where only first and third impulses entered the ventricles. Due to varying properties of AV node, conducting ratios may change (11).

This conduction mechanism is presented on ladder diagram (Fig. 7b).

\section{ECG elements overlapping}

The overlapping of high amplitude waves in classical isthmus-dependent AFL may reshape
Figure $7 b$. Excitation and conduction in atria, atrioventricular node and ventricles in atrial flutter with type A alternating Wenckebach phenomenon and $6: 2$ conduction.

High amplitude of $\mathrm{F}$ waves and low voltage of QRS complexes can seriously complicate the interpretation because of overlapping of ECG elements. At lead I (Fig. 7a) low amplitude QRS complexes can be seen. They are arrhythmic, although ordered in a particular regular sequence. In classical isthmus-dependent AFL, there are typical high amplitude F waves at II and III leads. They are overlapped with QRS complexes of low amplitude. If QRS complex coincides with the top of $\mathrm{F}$ wave, it is clearly visible. Conversely, when QRS coincides with the bottom of $F$ wave it's voltage is overshadowed that makes it less noticeable. At the first glance, the analysis of II and III leads on this ECG may seem to determine ventricular rate being equal to $55 \mathrm{bpm}$, while real ventricular rate is $110 \mathrm{bpm}$.

other ECG elements. Figure 8 shows a fragment of Holter monitoring. High amplitude $F$ waves 
were recorded in V3 and V5 modified bipolar chest leads. The positive part of these waves overlaps the beginning of ST segment making an impression of its elevation. The negative parts of $F$ waves overlaps $T$ waves end and this last part of $\mathrm{T}$ wave seems negative. Such ECG pattern is very similar to ECG in acute myocardial infarction with ST segment elevation. Nevertheless, the patient did not report angina pain, he felt quite well, though he reported the episodes of palpitations due to the atrial flutter.

\section{Atypical atrial flutter cases}

Atypical atrial flutter is a definition that covers different macro-reentrant atrial tachycardias that not pass through cavotricuspid isthmus. These tachycardias can be divided into three main groups: right atrium flutter that does not includes cavotricuspid isthmus, left atrium flutter (often pulmonary veins and mitral valve become a place of impulse circulation) and so called "incisional" flutters where macro-reentry mechanism occurs around iatrogenic scars in atria appeared after invasive procedures $(2,7)$. Diagnosis of the type of flutter using ECG characteristics is difficult if not impossible in patients that have previously undergone surgical "MAZE" ablation of the atria or percutaneous pulmonary vein isolation procedures. Multiple areas of slow conduction in these patients can result a high degree of variability of flutter wave morphology.

In case of the atypical AFL, ECG may contain slightly visible low amplitude $\mathrm{F}$ waves in leads II, III and aVF, while waves with a higher amplitude can be recorded in leads V1-V3 (Fig. 9.)

For 4:1 AV node conduction and normal ventricular rate, $F$ waves are discernible at isoelectric line between QRS complexes. In cases with faster ventricular rate and 2:1 AV conduction, diagnosis of atrial flutter with standard ECG is complicated and even sometimes impossible. In the case shown on Figure 10, atypical AFL must be thoroughly differentiated from sinus tachycardia. $\mathrm{F}$ waves characteristic for atrial flutter can be hardly seen only in V1-V3 leads.

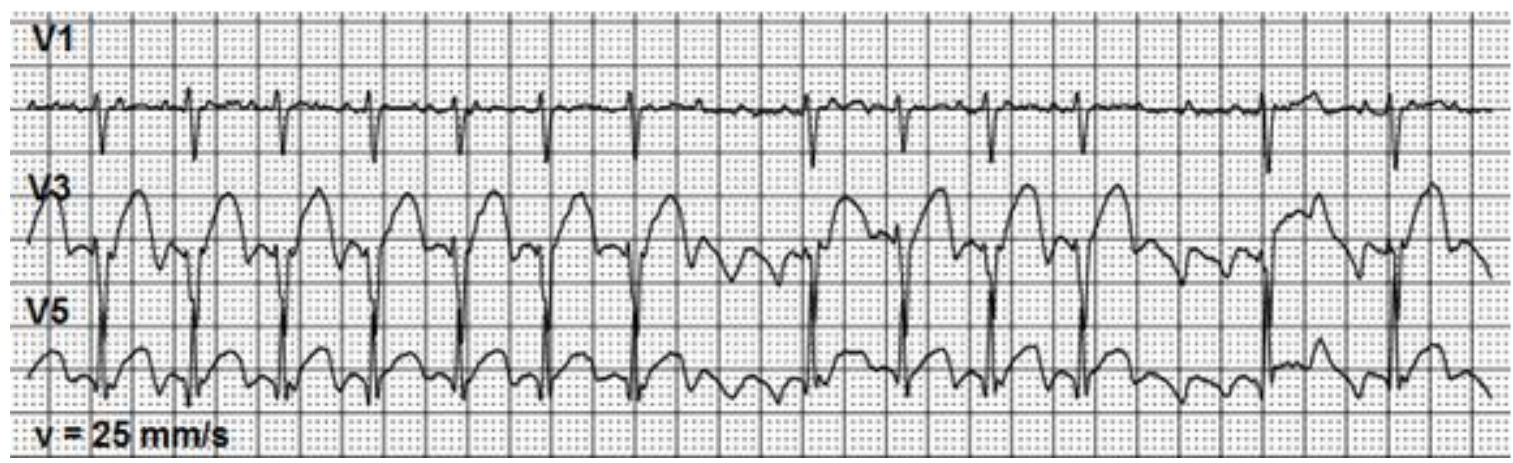

Figure 8. High amplitude waves of atrial flutter mimic the ST segment pathological elevation. 

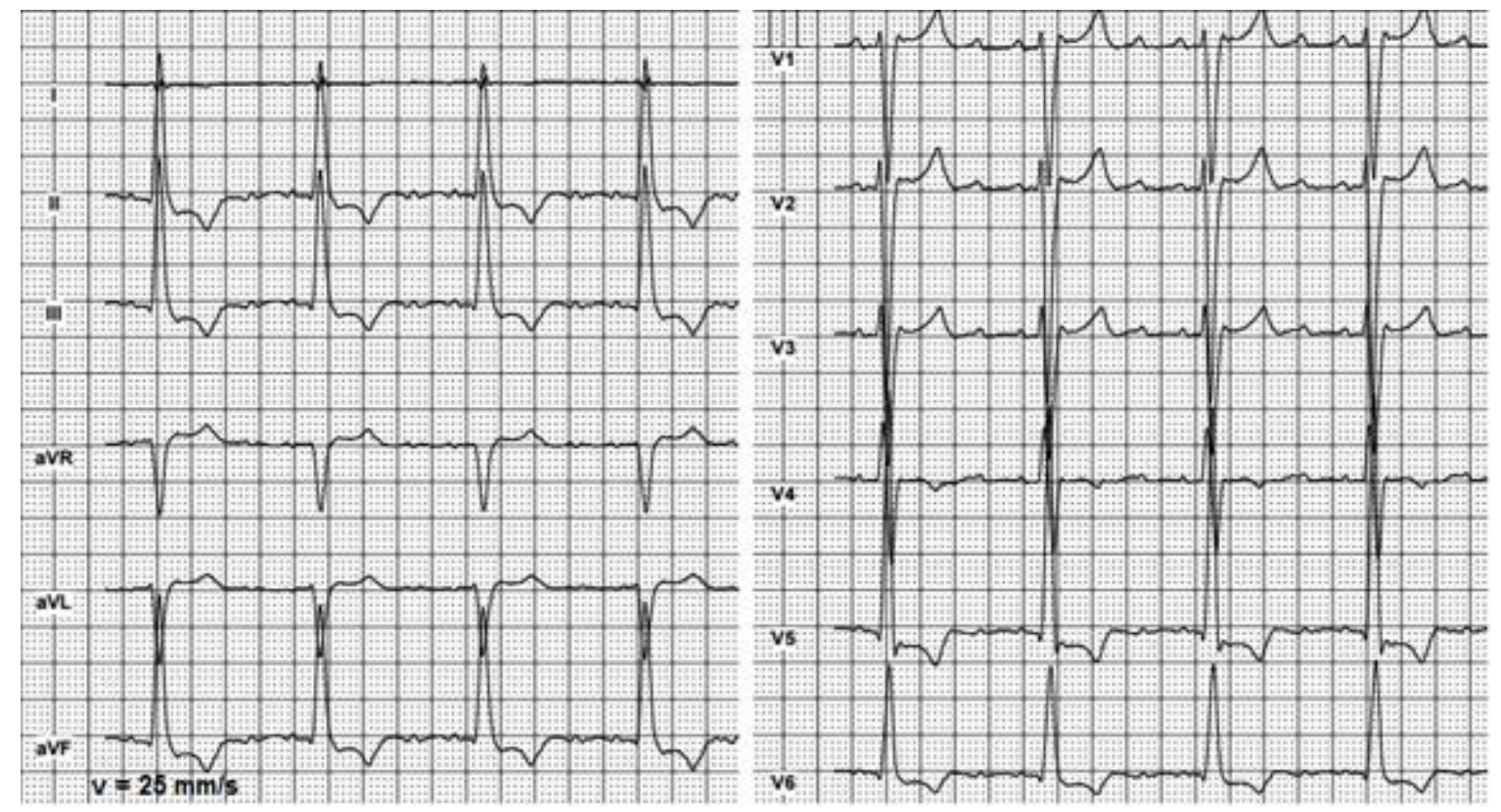

Figure 9. Atypical atrial flutter with a 4:1 conduction

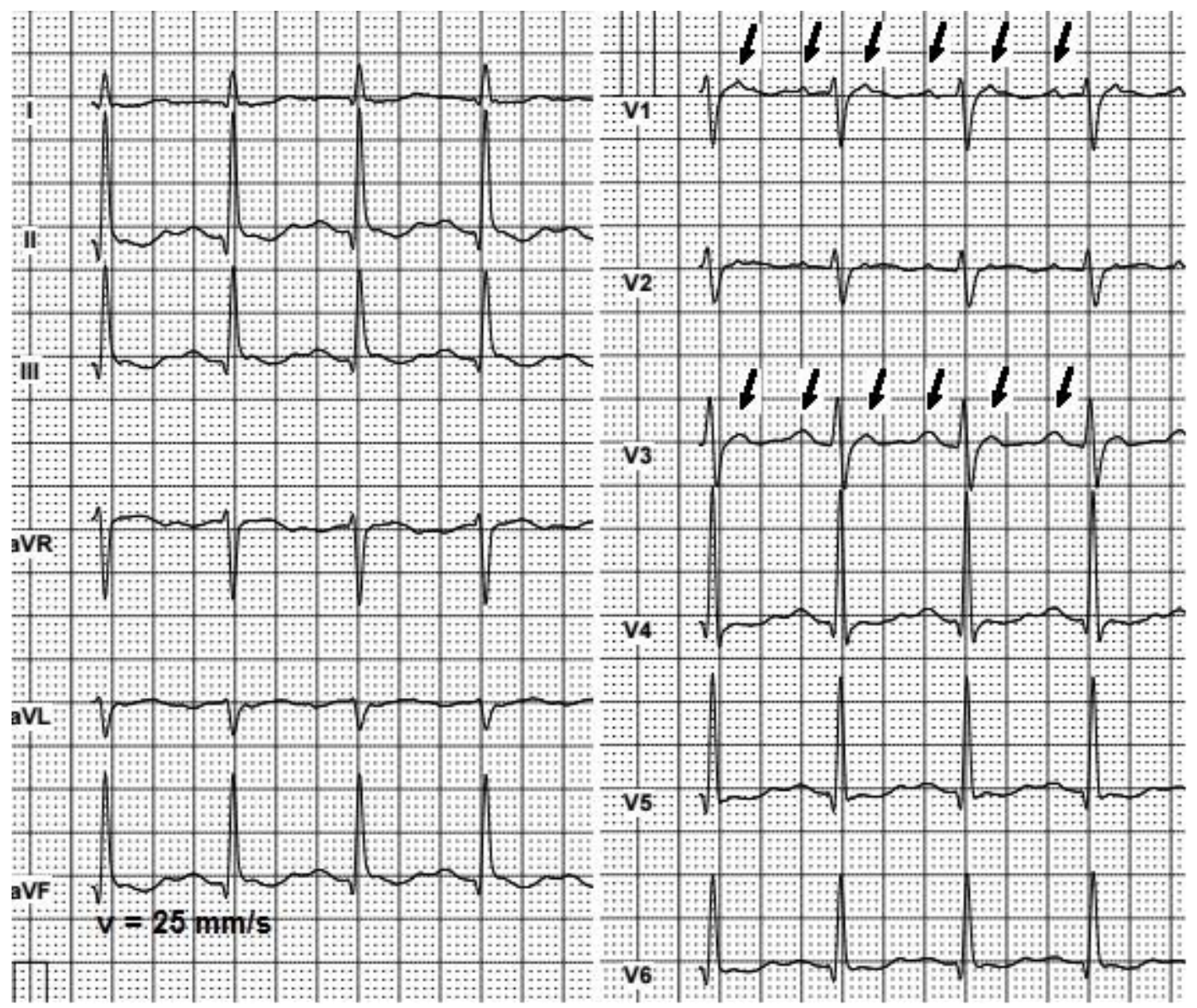

Figure 10. Atypical atrial flutter with 2:1 conduction. Arrows show F waves in leads V1 and V3. The highest amplitude of $\mathrm{F}$ waves in atypical atrial flutter is often recorded in lead V1. 


\section{Conclusion}

Atrial flutter is a heart rhythm disorder, which can be both easy and very difficult to interpret on standard 12-lead electrocardiogram. ECG data with presence of AFL may be diverse and often lead to diagnostic errors. Sometimes it is rather complicated to differentiate AFL from sinus tachycardia, other atrial reentrant tachycardia and atrial fibrillation. ECG findings must always be considered together with clinical data and results of other tests. Due to the increasing number of invasive cardiac procedures, including those for treatment of atrial fibrillation, the incidence of atypical, postoperative scar-related forms of atrial flutter is increasing. These atypical flutters can be especially challenging if not impossible to diagnose on the surface ECG. In these cases only an invasive electrophysiology study can provide the definite diagnosis.

Peer-review: external and internal

Authorship: M.S.S. and U.P.Ch-R. equally contributed to the preparation of manuscript and fulfilled authorship criteria.

Conflict of interest: No conflict of interests to be declared.

\section{References}

1. Saoudi N, Cosio F, Waldo A. Chen SA, Iesaka Y, Lesh M, et al. A classification of atrial flutter and regular atrial tachycardia according to electrophysiological mechanisms and anatomical bases. Eur Heart J 2001; 22: 1162-82.

2. Bun SS, Latcu DG, Marchlinski F, Saoudi N. Atrial flutter: more than just one of a kind. Eur Heart J 2015; 36: 2356-63.

3.Butta C, Tuttolomondo A, Giarrusso L, Pinto A. Electrocardiographic diagnosis of atrial tachycardia: classification, P-wave morphology, and differential diagnosis with other supraventricular tachycardias. Ann Noninvasive Electrocardiol 2015; 20: 314-27.

4. Goette A, Kalman JM, Aguinaga L, Akar J, Cabrera JA, Chen SA et al. HRA/HRS/APHRS/

SOLAECE expert consensus document on atrial cardiomyopathies: definition, characterization, and clinical implication. J Arrhythmia 2016; 32: 1247-8.
5.Kirchhof P, Benussi S, Kotecha D, Ahlsson A, Atar D, Casadei B, et. al. ESC Guidelines for the management of atrial fibrillation developed in collaboration with EACTS. Eur Heart J 2016; 37: 2893-962.

6.Waldo AL. The interrelationship between atrial fibrillation and atrial flutter. Prog Cardiovasc Dis 2005; 48: 41-56.

7. Doiny D, Merino JL. Atrial flutter: common and main atypical forms. E-Journal Cardiology Practice. 2013; 11: n14.

8. Kruse ML, Kruse JC, Leiria TL, Pires LM, Gensas CS, Gomes DG, et al. Relationship between silent atrial fibrillation and the maximum heart rate in the 24-hour Holter: cross-sectional study. Sao Paulo Med J 2014; 132: 359-63.

9.Peyrol M, Sbragia P, Bonello L, Lévy S, Paganelli F. Characteristics of isolated atrial flutter versus atrial flutter combined with atrial fibrillation. Arch Cardiovasc Dis 2011; 104: 5305 .

10. Page RL, Joglar JA, Caldwell MA, Calkins H, Conti JB, Deal BJ et al. 2015 ACC/AHA/HRS Guideline for the Management of Adult Patients with Supraventricular Tachycardia. J Am Coll Cardiol 2016; 67: 1575- 623.

11. Slama R, Leclerq J. F, Rosengarten $M$, Coumel P, Bouvrain Y. Multilevel block in the atrioventricular node during atrial tachycardia and flutter alternating with Wenckebach phenomenon. Br Heart J 1979; 42: 463-70. 